\title{
The Venus Life Equation
}

A White Paper for the Planetary Science and Astrobiology Decadal Survey 2023-2032

Noam R. Izenberg (noam.izenberg@jhuapl.edu, 443-778-7918, Johns Hopkins University Applied Physics Laboratory (JHUAPL), Laurel, Maryland, USA. )

Diana M. Gentry, NASA Ames Research Center, Moffett Field, CA, USA

David J. Smith, NASA Ames Research Center, Moffett Field, CA, USA

Martha S. Gilmore, Wesleyan University, Middletown, CT, USA

David Grinspoon, Planetary Science Institute, Washington, DC, USA

Mark A. Bullock, Science and Technology Corp., Boulder, CO, USA

Penelope J. Boston, NASA Ames Research Center, Moffett Field, CA, USA

Grzegorz P. Slowik, University of Zielona Góra, Poland

\section{Co-signers with their respective institutions:}

Jennifer Whitten (Tulane University)

Darby Dyar (Planetary Science Institute)

Constantine Tsang (Southwest Research Institute)

Paul Byrne (North Carolina State University)

Jaime Cordova (University of Wisconsin-Madison)

Kevin Baines (Jet Propulsion Laboratory)

Timothy N. Titus (US Geological Survey)

Stephen Kane (University of California, Riverside)

Jorn Helbert (DLR - German Aerospace Center)
Pat Beauchamp (Jet Propulsion Laboratory)

Michael Way (NASA Goddard Institute for Space Studies)

Rakesh Mogul (California State Polytechnic

University-Pomona)

Julie Nekola Novakova (European Astrobiology Institute)

Kandis-Lea Jessup (Southwest Research Institute)

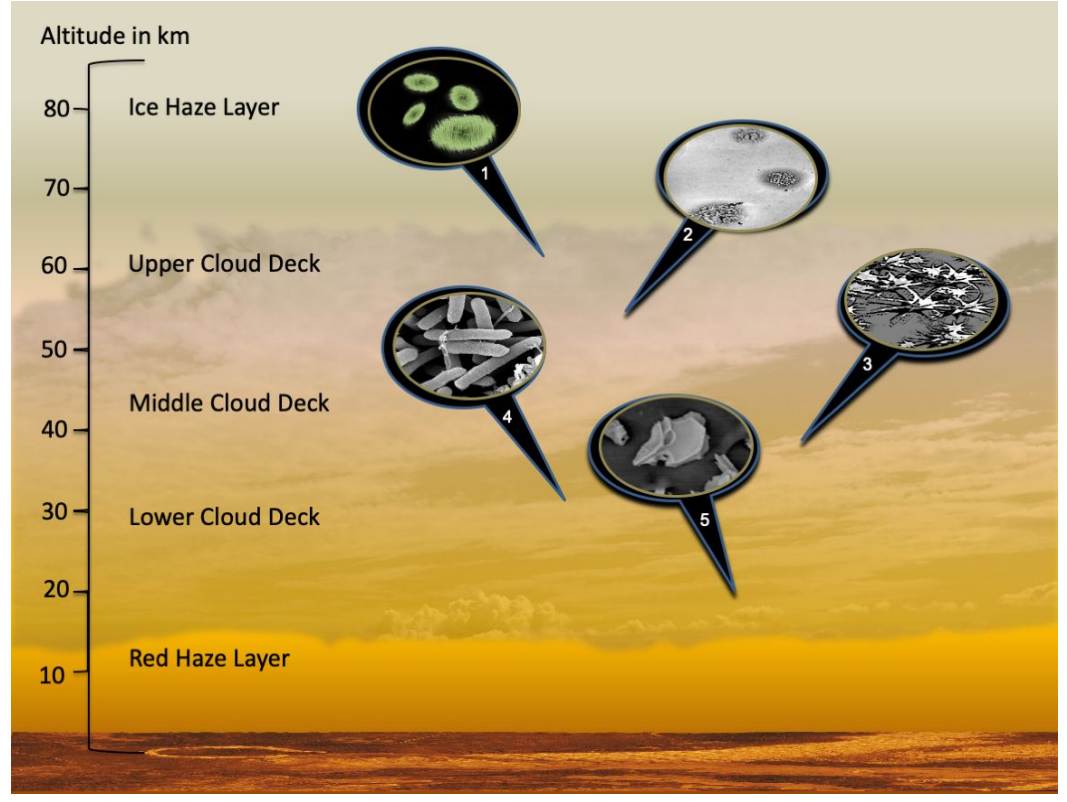

Frontispiece: Notional particles potentially to be encountered in the Venus cloud decks, inspired by terrestrial atmospheric sampling, to guide future instrument and analysis selection: 1) Complex shapes with fluorescent properties, 2) Particulate aggregates of sulfates and related compounds, 3) Unidentified group of complex shapes adhered to an aerosol particle, 4) Objects that resemble Earth bacteria or archaea, and 5) volcanic ash particles. 


\section{Key Points}

- The chance of extant life in the clouds of Venus is estimable from what we know about Life on Earth and the history of Venus.

-We define and consider Origination, Robustness, and Continuity factors, and conclude that the chance life exists on Venus today is non-zero.

- Better understanding of Venus' history and current conditions through new missions including in-situ investigations would constrain the Venus Life Equation, even in absence of direct detection of life.

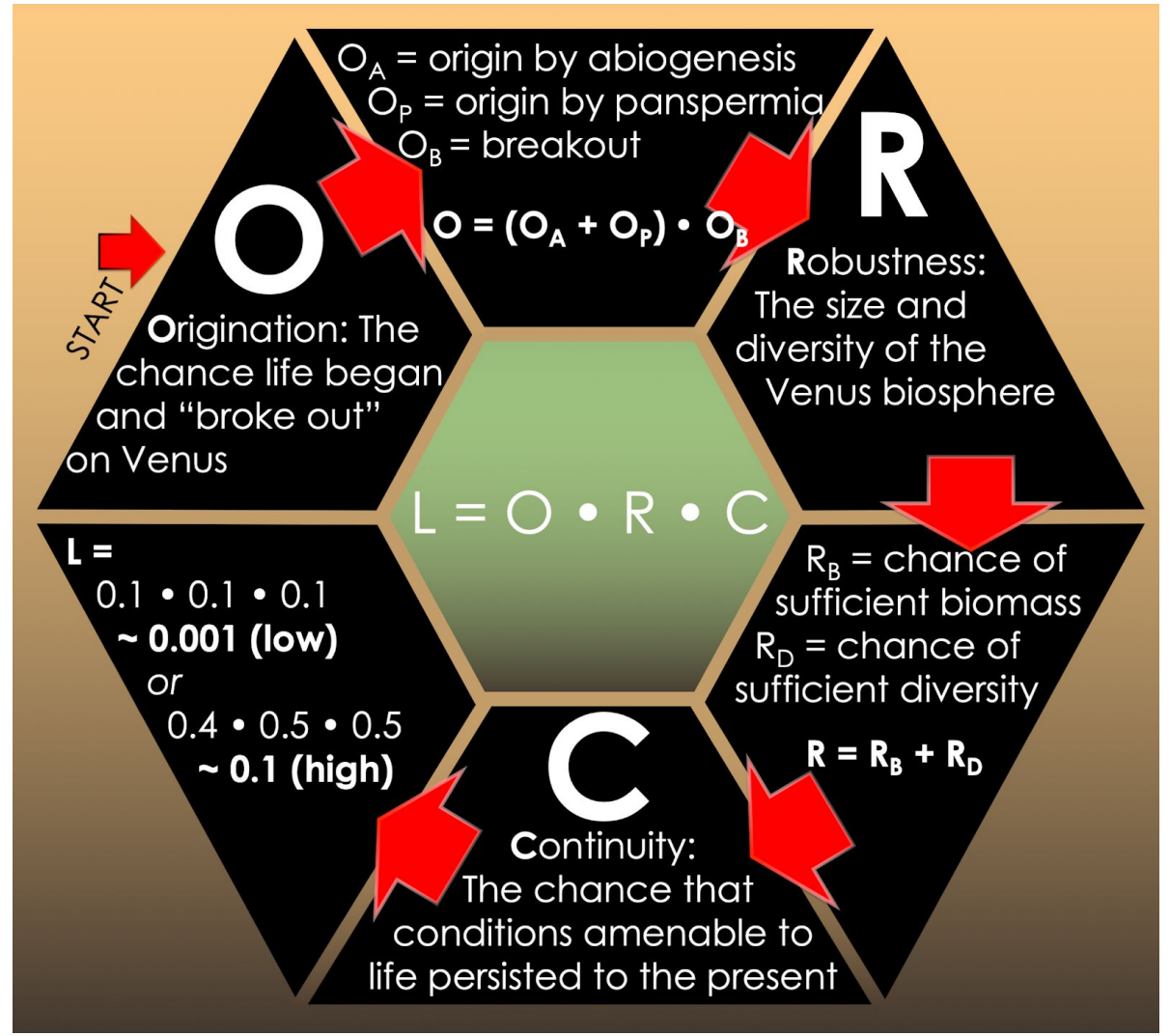

Figure 1. Schematic of the Venus Life

Equation. Variables and equations elaborated in text. The final estimate for $L$ in this figure represents an example low and high range of estimates for each factor.

Introduction: One of the biggest motivations for exploring the solar system beyond Earth is examining whether extant life currently exists, or now-extinct life once existed, on worlds beyond ours. Our current state of knowledge of the past and present climate of Venus suggests it once had an extended period - perhaps 2 billion years where a water ocean - and a land-ocean interface - could have existed on the surface, in conditions resembling those of Archaean Earth $[1,2]$. Although today Venus' surface $\left(450^{\circ} \mathrm{C}, 90\right.$ bars $)$ is not hospitable to life as we know it, there is a zone of the Venus middle atmosphere, at around $55 \mathrm{~km}$ altitude, just above the sulfuric acid cloud layer, where the conditions are reasonably Earth-like [3]. The question of whether life could have - or could still - exist on the Earth's closest neighbor is in fact more open today than it's ever been [4-6]. This paper approaches the question of extant life on Venus in a similar manner as Drake Equation [7]. We approach the question of whether life exists currently in the Venus atmosphere as an exercise in informal probability - seeking qualitatively the likelihood of the answer being nonzero. 
The Venus Life Equation: The Venus Life Equation (Figure 1) is expressed as:

$$
\mathbf{L}=\mathbf{O} \cdot \mathbf{R} \cdot \mathbf{C}
$$

where $L$ is the probability (zero to 1 ) of there being life currently in a sustained airborne Venus ecosystem, $O$ (origination) is the probability life ever began on Venus and survived long-term, $R$ (robustness) is the potential current and historical size and diversity of the Venus biosphere, and $C$ (continuity) is the probability that conditions amenable to life persisted spatially and temporally to the present. The following sections describe each variable.

Origination: Life on a planet can start via independent abiogenesis, or importation from elsewhere (panspermia), where:

$$
\mathbf{O}=\mathbf{O}_{\mathrm{A}}+\mathbf{O}_{\mathrm{P}} \quad \text { or possibly: } \quad \mathbf{O}=\mathbf{1}-\left(\left(\mathbf{1}-\mathbf{O}_{\mathrm{A}}\right) \cdot\left(\mathbf{1}-\mathbf{O}_{\mathrm{P}}\right)\right)
$$

where $\mathrm{O}_{\mathrm{A}}$ is the likelihood of origin by abiogenesis and $\mathrm{O}_{\mathrm{P}}$ is the likelihood of origin by panspermia. The difference between Eqns. 2 and 3 is that Eqn. 3 removes a potential double-counting if life has arisen by both abiogenesis and panspermia, i.e., the probability of two separate geneses. If $\mathrm{O}_{\mathrm{A}}$ and $\mathrm{O}_{\mathrm{P}}$ are both small, this difference is negligible.

$\mathrm{O}_{\mathrm{A}}$ depends on how likely it is for life to arise independently. In our solar system, empirically, we assign $\mathrm{O}_{\mathrm{A}} \sim 1$ for Earth. For other bodies, for lack of other definitive evidence, we assume the baseline is effectively zero, where $\mathrm{O}_{\mathrm{A}}$ increases if current or historical conditions exist that are similar to those of early Earth. $\mathrm{O}_{\mathrm{P}}$ in our solar system may be nonzero from possible transportation of life due to impacts emanating from Earth, at least [8, 9]. Because of its relative proximity and size, Venus is the most likely to receive viable life from Earth [10], and thus, over geologic history, we can estimate a value of $\mathrm{O}_{\mathrm{P}} \sim 0.1-0.5$. But just starting life would not be enough to result in a sustained biosphere.

For the purposes of life that we might be able to detect with astrobiological investigations, breakout, $\mathrm{O}_{\mathrm{B}}$, is an essential component of origination. It is the chance life escaped its point of origin to spread across the planet. On Earth life may have arisen once, or dozens or thousands of times in different surface or subsurface regions only to be snuffed out by events for which we have no record. This is encapsulated in the variable $\mathrm{O}_{\mathrm{B}}$, which for Earth became 1 early in its history. If we assume early Venus was similar to Archean Earth for 2+ billion years, an $\mathrm{O}_{\mathrm{B}}$ of 0.9 to 1 for Venus may be reasonable.

Accounting for breakout modifies the Origination term:

$$
O=\left(O_{A}+O_{P}\right) \cdot O_{B} \quad \text { or } \quad O=\left(1-\left(\left(1-O_{A}\right) \cdot\left(1-O_{P}\right)\right)\right) \cdot O_{B}
$$

On Venus, example estimates for the subfactors might range from $\leq 0.1$ to 0.4 as the probability span for life getting a foothold on the second planet.

Why $O$ is not zero for Venus: Current models suggest that early Venus conditions paralleled those of early Earth during the period in which Earth life arose [1, 2], which, absent other information, supports an Earth-like value of $\mathrm{O}_{\mathrm{A}} 0.9 \sim 1$. Regardless of a potential independent biogenesis on Venus, we know that endolithic terrestrial microbes have been sent towards Venus throughout its habitable history, yielding an estimate of $\mathrm{O}_{\mathrm{P}} \sim 0.1-0.5$. 
Robustness: Life on Earth has survived in part because it spread so widely, with great variety and quantity; it was therefore hard to completely eradicate during acute or gradual environmental changes. An estimation of this robustness may be expressed with:

$$
\mathbf{R}=\mathbf{R}_{\mathbf{B}} \cdot \mathbf{R}_{\mathbf{D}}
$$

where $R_{B}$ is a measure of potential biomass supported over time, and $R_{D}$ is a similar measure of potential diversity.

Planetary and Astrobiology Study of Robustness: Like O, R is affected by the " $\mathrm{n}=1$ " problem. Earth, past and present, is our only example of a biosphere. We can estimate how much lower an Earth-like planet's R could be under less-favorable global conditions using results from studies of life in extreme environments, comparative ecology, paleoclimatology, etc. However, it is difficult to make conservative estimates about a planet theoretically more habitable than Earth. To account for these limits, we define $R$ here as a fraction of $R_{\text {Earth }}$, and let $R_{\text {Earth }}=1$. This makes our bias explicit, and allows a straightforward recalculation if one wishes to make different assumptions about Earth's relative habitability.

Planet-scale biomass on Earth is usually quantified as either organically bound carbon (Gt C) or primary production $\left(\mathrm{Gt} \mathrm{C} \cdot \mathrm{yr}^{-1}\right)$. Both measures assume a biosphere based on carbon, and neither are necessarily true for other potential biospheres [11]. However, part of the astrobiological appeal of Venus is its similarity to Earth during the early period in which life may have arisen, implying a similar potential biochemistry. We therefore use estimates of Earth's $R_{B}$ in $\mathrm{Gt} \mathrm{C}$ as a baseline. Early Venus may have been relatively similar to early Earth, so we use early Earth as a template for estimating a "best case" $\mathrm{R}_{\mathrm{B}}$ for early Venus. Some estimates for Earth's Archaean biosphere place it in the range of 100 - 400 Gt C [12], about $0.2-0.7$ times Earth's current biomass ( 550 Gt C) [13].

Though much remains unknown about Venus's past history, it is clear that an early state modeled to have clement oceans is very different from its current state, in which liquid water cannot exist on the surface and the most commonly suggested extant habitat is aerosols within certain bands of the cloud layers. We know very little about the possible productivity of a modern Venus ecosystem, although some potential nutrient sources have been proposed [5, 14].

On Earth, biomass over time has been sufficient to allow survival despite dramatic climatic change and near-global extinction events. The most commonly argued case for life on modern-day Venus is that life, whatever its origin, could have colonized the dense, persistent cloud layer and endured there after the loss of the surface oceans [15]. However, Earth has no habitat directly analogous to Venus's cloud layer. The closest regime, in terms of chemistry and isolation from surface nutrient and water sources, is probably the stratospheric sulfate layer [16], where the longest-enduring microbe-sized aerosols may have residence times of years. However, under stratospheric conditions it is likely impossible for terrestrial microorganisms to metabolize, grow, or reproduce. Survivors recovered at extreme heights above the Earth's surface tend to be dormant, resilient, endospore-forming bacteria enduring harsh irradiation until dropping out by gravitational settling [17]. Additional in-situ cloud microbiology measurements will be required 
before it can be determined if some life on Earth is periodically "independent" of the planet's surface $[18,19]$.

We can place an order-of-magnitude upper bound on the present-day $R_{B}$ estimate using a similar approach. Let's assume that all the particles in Venus's cloud layer larger than $0.2 \mu \mathrm{m}$, the lower end of terrestrial microbes' size range, are putative microorganisms. A quick calculation using reported particle concentrations [20] yields a count of $5 \times 10^{24}$ potential organisms. By comparison, the estimated number of prokaryotes on Earth is in the range of $4 \times 10^{30}$ [21]. These assumptions yield an upper limit on the current $R_{B}$ of Venus of $\sim 0.000001$. Other suggestions that have been put forward for Venus include surface life adapted to use supercritical carbon dioxide as a solvent [22], and subsurface microbes in refugia of highly pressurized water [23]. $R_{B}$ can be recalculated considering these farther-ranging hypotheticals to produce a different answer.

Life on Earth, which represents our baseline $\mathrm{R}_{\mathrm{D}} \sim 1$, is incredibly diverse, where nearly every liquid and solid surface measured to date is colonized with a well-developed ecosystem. Even more so than with biomass, there are a wide variety of biodiversity metrics in use, and each reflects one or more terrestrial biases. More quantitative metrics, such as taxonomic diversity, are also less extensible to other theoretical biospheres. Functional diversity, which reflects how many distinct niches life can occupy in a given habitat, is probably the most intuitively applicable to a theoretical non-terrestrial biosphere. As with biomass, the metric chosen should reflect constrainable similarities between Earth and Venus.

We know very little about the biodiversity of Archaean Earth, even as an analogue. For hundreds of millions of years, early Earth lacked several major functional niches present today [24], including oxygenic photosynthesis and all, or nearly all, land-based ecology. We have no way of knowing how many historical niches may once have existed - e.g., chemolithotrophs utilizing minerals only stable in a reducing atmosphere - but are now lost. We know even less about early Venus. However, since $\mathrm{R}$ is meant to represent a best-case, and we know that any extant life on Venus is likely to be a relic of an earlier more thriving era, we can pick an example range for early Earth's $R_{D}$ of $0.1-0.5$ and use this as our estimate for early Venus as well.

Modern-day Venus is a more tractable case. Although, as with $R_{B}$, there is no direct analogue, we can estimate an upper bound based on partial analogue reasoning. Chemically speaking, terrestrial acid hot springs have been proposed as the inhabited environments that most closely reproduce Venus cloud temperature $\left(97--45^{\circ} \mathrm{C}\right)$ and $\mathrm{pH}$ (less than -1.3 to 0.35$)[15,25]$, whereas deserts or concentrated brines may best represent the low water activity in Venus aerosols ( $\sim 0.02$ at a relatively optimistic assumption of $75 \% \mathrm{H}_{2} \mathrm{SO}_{4}$ and $\left.25 \% \mathrm{H}_{2} \mathrm{O}\right)$ [26-29]. Each of these environments show significantly less diversity than more typical mesophilic environments. At $\mathrm{pH}$ levels at or below 1, terrestrial life is limited to a few lineages of archaea [30]. Brines at water activities below 0.75 are similarly limited to other lineages of archaea [31, 32]. Life forms in both environments are restricted in the metabolic pathways which occur [30, 32]. Only the most extreme deserts on Earth approach water activities below 0.1, and here, 
though more taxonomically diverse, life is primarily phototrophic, adapted to long periods of inactive desiccation followed by brief bursts of activity during sporadic water influx.

Why $R$ Is Not Zero for Venus: Because $R$ represents a "best-case" biosphere, it could only be zero for a target environment which meets no known criteria for habitability -- for example, the sun, dry lunar regolith, or the exposed surface of an asteroid. While the potentially supportable biosphere on modern Venus may be quite low or limited by terrestrial standards, the relative clemency of early Venus, and its similarity to the empirically inhabited early Earth, means R should not be zero.

Continuity: This factor reflects the necessity of continuous existence of habitats over time and space; or, equivalently, the lack of global extinction events. Environmental continuity is affected by both internal and external factors. The former includes variations in the carbonate/water cycles that are governed by plate recycling, rates of volcanism and rock weathering. Study of the composition and deformation histories of the most ancient terrains on Venus (tesserae) may help determine the presence, extent and duration of some of these factors [e.g. 33]. External factors include solar-system wide events such as stellar variability (life-threatening flares/coronal mass ejections), stellar aging (changing luminosity inducing climatic shifts), and large impactors [34, 35]. Some of these external factors, such as stellar lifetime, are empirically known to be 1 for Venus by the continuing presence of life on neighboring Earth. Others, such as activity sufficient to sterilize life only out to $0.8 \mathrm{AU}$, or an extinction-level CME while Venus and Earth were in opposition, must be estimated.

Continuity can be quantifiably constrained for Venus through direct measurement of the availability of current resources in potential habitats (e.g. the elements $\mathrm{C}, \mathrm{H}, \mathrm{N}, \mathrm{O}, \mathrm{P}, \mathrm{\&} \mathrm{S}$ and solvents as necessary building blocks for Earth-like biology in Venus clouds), and through unraveling the geologic history of the planet to determine if a continuous path might have been available for life to evolve to survive and maintain itself for tens or hundreds of millions of years of post-ocean Venus history. For example, one possible pathway to extant Venus life would require conditions to evolve contiguously and continuously from a marine-land interface to a globe-spanning biosphere, to eventual adaptation towards complete airborne life cycles and a biosphere maintainable solely in the clouds.

Complications for continuity are rooted in both understanding of terrestrial biology and lack of understanding of Venus' geologic history and present conditions. If one assumes a "terrestrial-like" biochemistry, neither the trace composition of Venus's aerosols nor current conditions such as atmospheric circulation of dust are understood well enough to determine water activity or the presence of bioavailable forms of nitrogen and phosphorus, let alone the more rare 'essential' heavy elements like $\mathrm{Fe}, \mathrm{Zn}, \mathrm{Pb}, \mathrm{Cu}, \mathrm{Sn}, \mathrm{V}, \mathrm{Cd}, \mathrm{Ni}, \mathrm{Se}, \mathrm{Mn}, \mathrm{Co}, \mathrm{Cr}$, As, Mo and $\mathrm{W}$. Detections of both phosphorus and iron were reported by the Vega X-ray fluorescence radiometers [36], but a comprehensive trace elemental assay of the Venus clouds has not been performed. Also, what is observed to be an "essential" element in terrestrial biota is the product of an opportunistic evolutionary process which might well have found "work arounds" in other 
planetary environments with a different complement of available elements. More exotic proposed biochemistries (such as direct use of sulfuric acid as an alternative polar solvent) are even less constrained in terms of energy and chemistry requirements [37, 38].

Non-chemical requirements are also critical. Energy pathways such as photosynthesis or chemosynthesis need to have been established and maintained or evolved to in a similar contiguous and continuous manner. For example, although Venus receives more photonic energy at the top of its atmosphere than Earth, the thick atmosphere and haze layer reflect or block larger fractions of it. This is particularly important for a potential atmospheric ecosystem, as the residence time of potential aerosol habitats imposes a particular constraint. Many terrestrial microbes in extremely harsh or nutrient-limited environments have generation times of weeks to months, potentially in combination with long periods of inactivity. If microbe-bearing aerosols, on average, settle out (as on Earth) or fall to an altitude at which they dry out or boil off (as on Venus) faster than the microbes can reproduce, an aerosol-based biosphere without periodic injections from other reservoir habitats will not be stable over the long term, even if short-term conditions are otherwise favorable.

One of the major sub-factors of $\mathrm{C}$ specific to Venus is the timeline of Venus's water loss and cloud formation. Although life is capable of very rapid adaptation and diversification in some circumstances, major habitat transitions on Earth such as colonization of land took at least hundreds of millions of years. The shorter the period of overlap between the potential origination of life in the oceans and the formation of the modern-day habitat of persistent cloud cover, the lower the likelihood of colonization; and if the two did not overlap at all, it would be negligible. Though this timeline is not currently well constrained, there may have been a gap between the end of Venus' surface oceans and the current thick cloud deck of 10s Myr to 100s Myr [2].

Most of the areas delineated in the Goals, Objectives, and Investigations for Venus Exploration [39] document will result in direct quantitative improvement of the estimate for C. The entirety of Goal 1, in fact, prioritizes the understanding of Venus' early history and potential habitability.

Why C Is Not Zero for Venus. At the moment, this could be the most difficult stipulation of the Venus Life Equation. We simply don't know enough about Venus' evolution to do more than make models and geologically and evolutionary plausible 'what if' scenarios. Assigning for $\mathrm{C}$ a range of 0.1 to 0.5 may be generous, or not. Continuity estimates can only be vastly improved with each new mission we send to Venus.

Life: With estimates for all 3 factors for Venus, we can calculate a range for the chance of life. Using example low and high values throughout:

$$
\mathbf{L}=\mathbf{0 . 1} \cdot \mathbf{0 . 1} \cdot \mathbf{0 . 1}=\mathbf{0 . 0 0 1}(\mathrm{low}) \quad \text { or } \quad \mathbf{L}=\mathbf{0 . 4} \cdot \mathbf{0 . 5} \cdot \mathbf{0 . 5}=\mathbf{0 . 1} \text { (high) }
$$

These numbers are simply example estimates. Individual investigations and additional constraints, experiments, or observations may drive any one of the factors or subfactors higher or lower. 
Beyond Venus: This exercise can be performed for any potential abode of life in our solar system and adapted and estimated for any potential habitable world. The L determined by the Venus Life Equation, adapted for and integrated over many possible worlds, is related to the term $f_{1}$ of the Drake Equation [7]: the fraction of planets in our galaxy that develop life. The equation applied to Venus shows how we might approach questions of habitability on worlds beyond Earth.

Consequences for Planetary Protection: Currently, NASA classifies Venus missions under planetary protection Category II [40]. The Category II classification of the NAS study remains unchallenged by a nonzero value for L. Like any life detection experiment, however, any in-situ instrumentation will need to invoke a high level of internal protection to ensure accurate measurement.

Conclusion: We find the probability for extant life on Venus is non-zero. The study of Venus is critical to address the strategic objective of the Planetary Science Division to determine the potential for life elsewhere. This argues strongly for exploratory missions operating within the cloud environment as part of any comprehensive strategy to look for extant life in the solar system.

References: [1] Way et al., Geoph Res Lett 2016;43(16) 8376-8383; [2] Del Genio and Way, J Geophys Res Planets 2020;125(5):e2019JE006276; [3] Cavicchioli, . Astrobiology 2002;2,3:281-292; [4] Morowitz and Sagan, . Nature 1967;215:1259-1260; [5] Limaye et al., Astrobiology 2018;18(9):1181-98; [6] Seager et al., Astrobiology, 2020; in press; [7] Burchel, Int J Astrobio 2006;5(3):243-250; [8] Nicholson, Trends Microbio 2009;17(6):243-350; [9] Beech et al., Am J Astron Astroph 2018;6(3): 81-90; [10] Gladman et al., Science 1996;271:1387-1392; [11] NRC, Limits of Organic Life Natl Acad Press 2007; [12] Schulze-Makuch et al., Astrobiology 2004;4(1): 11-18; [13] Franck et al., Biogeosci Dsc 2005;2(6): 1665-79; [14] Grinspoon and Bullock, Geophys Mon Ser 2007;176:191-206; [15] Bar-On et al,. 2018 P Natl Acad Sci 2018;115 (25):6506-11; [16] Gentry and Dahlgren, Venera-D Landing Sites \& Cloud Hab Wkshp 2019: Moscow, Russia; [17] Bryan et al., ISME Journal 2018;13,11:2789-2799; [18] Amato et al., Scientific Reports 2019;9(1):4383; [19] Smith, Astrobiology 2013;13(10): 981-990; [20] Esposito et al., in Venus, Univ. Ariz. Press, 1984;16:484-564; [21] Whitman et al., P Natl Acad Sci 1998;95(12): 6578-6583; [22] Budisa and Schulze-Makuch, Life 2014;4 (3):331-40; [23] Schulze-Makuch et al., Astrobiology 2005;5(6):778-95; [24] Nisbet, Geol Soc, London, Sp Pub 1995;95(1):27-51; [25] Krasnopolsky, Spect. and Photochem Planet Atmo Iono. Cambridge,2019;243; [26] Deno and Taft, J Am Chem Soc 1954;76(1):244-248; [27] Hansen and Hovenier, J Atmo Sci 1974;31:1137-1160; [28] Kleft, Encyc of Env Microbio Wiley 2003;1-25. [29] Bolhuis et al., BMC Genomics 2006;7(1),1; [30] Barrie Johnson and Hallberg, Adv Microb Physiol 2008;54:201-55; [31] Grant, Phil Trans Royal Soc Bio Sci 2004;359(1448):1249-67; [32] Oren, Extremophiles Handbook 2011;1:344-6; [33] Gilmore et al., Space Sci. Rev., 2017;11:(1-30); [34] Bostrom and Circovic, Global catastrophic risks. 2011 Oxford University Press; [35] Chapman and Morrison, Cosmic catastrophes. 2013 Springer; [36] Andreychikov et al., Cosmic Res 1987;25:16; [37] Schulze-Makuch and Irwin, Life in the Universe 2008;7-24. Springer; [38] Cockell and Nixon, Planet Space Sci 1999;47(12):1487-1501; [39] VEXAG, GOI 2019 www.lpi.usra.edu/vexag/reports/VEXAG_Venus_GOI_Current.pdf; [40] NAS Planetary Protection National Academies Press, 2006;16p. 